\title{
ERHÖHTES HERZ-KREISLAUF-RISIKO?
}

\section{Kalziumpräparate erneut in der Diskussion}

Bereits im letzten Jahr wurde in einer Metaanalyse ein Zusammenhang zwischen einer Kalziumsupplementierung und einem erhöhtem Herzinfarktrisiko festgestellt. Diese Beobachtung stand bisher im Widerspruch zu Daten aus der großen WHI-Studie. Eine Re-Analyse der WHI-Daten (unter Ausschluss von Frauen, die schon vor Studienstart Kalzium einnahmen) zeigt aber auch hier eine leichte Erhöhung des kardiovaskulären Risikos unter Kalzium plus Vitamin D. Auch in einer neuen umfassenderen Metaanalyse mit 28000 Patienten ist die
Supplementierung von Kalzium, allein oder zusammen mit Vitamin $D$, mit einem um etwa $20 \%$ erhöhten Risiko für Herzinfarkt und Schlaganfall verbunden. Die Studienautoren fordern daher eine Neubewertung der Osteoporoseprävention mit Kalzium. Nach Einschätzung der BMJ-Kommentatoren ist das jedoch verfrüht. Zumindest die Kombination aus Kalzium und Vitamin $D$ als Ergänzung einer Bisphosphonattherapie sei kardiovaskulär sicher.

Bolland MJ et al. BMJ 2011;342:d2040; Abrahamsen B, Sahota O. BMJ 2011;342:d2080

\section{THERAPIEEMPFEHLUNG FÜR PATIENTEN}

\section{Würden Sie für sich selbst genauso entscheiden?}

Ein Patient hat die schwierige Wahl zwischen zwei Behandlungsalternativen: Die Therapie, mit der seine Überlebenschancen höher sind, kann unangenehme Nebenwirkungen zur Folge haben. Wozu würden Sie als Arzt ihm raten? Und welche Behandlung würden Sie wählen, wenn Sie selbst erkrankt wären?

Das ist offenbar nicht immer dasselbe, wie die Befragung von 750 Hausärzten ergab: Bei einem Kolonkarziom würden 37,8\% der Ärzte für sich selbst die Behandlung mit der höheren Mortalität wählen, aber nur 24,5\% würden diese Behandlung ihren Patienten

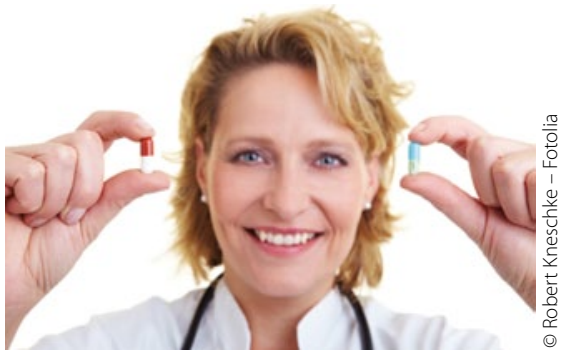

empfehlen. Auch im Fall einer Vogelgrippe nannten die Ärzte das prognostisch weniger günstige Medikament häufiger für die Selbsttherapie (62,9\% vs. $48,5 \%)$.

Ubel PA et al. Arch Intern Med 2011;171(7):630-634

\section{UNFALLFORSCHUNG}

\section{Warum es bei Teenagern öfter kracht}

Junge Führerscheinneulinge verursachen so oft tödliche Unfälle wie erwachsene Fahrer. In einer US-Studie wurde jetzt nach den Ursachen geforscht. Untersucht wurden 800 Unfälle, an denen jugendliche Fahrer beteiligt waren.

In $21 \%$ der Fälle hatten die Fahrer Gefahrensituationen nicht erkannt. Weitere 21\% waren zu schnell unterwegs, und bei $20 \%$ der Unfälle war der Fahrer durch Vorgänge innerhalb oder außerhalb seines Wagens abgelenkt. Für Fahrneulinge ist es typisch, dass sie ihre Aufmerksamkeit vor allem auf Vorgänge richten, die sich mehr oder weniger direkt vor der Motorhaube ihres Wagens abspielen.

Accident Analysis \& Prevention, 2010; DOI: 10.1016/j. aap.2010.10.019

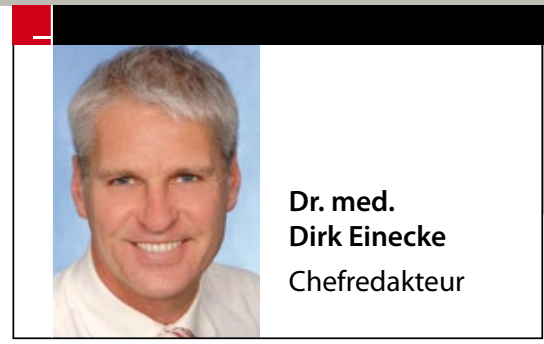

PROGNOSEFAKTOR

\section{Shoppen hält am Leben}

Wenn alte Menschen täglich Einkäufe machen, dann ist das ein gutes Zeichen: Ihr Risiko, in den nächsten zehn Jahren zu sterben, ist nur halb so hoch wie bei Senioren, die selten oder nie einkaufen gehen. Selbst wenn in der kognitiven und physischen Fitness kein Unterschied besteht, haben VielEinkäufer ein geringeres Mortalitätsrisiko (-27\%) als Wenig-Einkäufer. Möglicherweise ist ihr Konsumverhalten also nicht nur ein Indikator für ihre Lebenserwartung, sondern wirkt sogar lebensverlängernd.

Chang Y-H et al. J Epidemiol Community Health, April 2011, online first; doi:10.1136/jech.2010.126698

\section{ERNÄHRUNGSFORSCHUNG}

\section{Wer Dicke sieht, isst mehr}

Wer wert auf seine schlanke Linie legt, sollte möglicherweise nicht nur darauf achten, was er isst, sondern auch mit wem er speist.

In einer Sudie nahmen sich Probanden, die ein Foto mit einer übergewichtigen Person sahen, doppelt so viele Bonbons wie Probanden, denen Bilder einer normalgewichtigen Person vorgelegt wurde. Auch in einem Kekstest wurde die doppelte Menge verzehrt, wenn eine übergewichtige Person zugegen war. Selbst Probanden, die angaben, auf ihre schlanke Linie zu achten, langten beim Anblick Übergewichtiger kräftiger zu.

Journal of Consumer Research, October 2011 (published online March 17, 2011) DOl: 10.1086/659754 